

Chamfer metrics in mathematical morphology

P.F.M. Nacken

Department of Operations Research, Statistics, and System Theory

Reporf BS-R9309 April 1993 
$\mathrm{CWI}$ is the National Research Institute for Mathematics and Computer Science. CWI is part of the Stichting Mathematisch Centrum (SMC), the Dutch foundation for promotion of mathematics and computer science and their applications. SMC is sponsored by the Netherlands Organization for Scientific Research (NWO). CWI is a member of ERCIM, the European Research Consortium for Informatics and Mathematics.

Copyright (C) Stichting Mathematisch Centrum P.O. Box 4079, 1009 AB Amsterdam (NL) Kruislaan 413, 1098 SJ Amsterdam (NL)

Telephone +31205929333

Telefax +31205924199 


\title{
Chamfer Metrics in Mathematical Morphology
}

\author{
P.F.M. Nacken \\ Institute for Perception TNO \\ Kampweg 5, 3769 DE Soesterberg, The Netherlands
}

\begin{abstract}
In this paper, an integration of chamfer metrics into mathematical morphology is presented. As chamfer metrics can approximate the Euclidean metric accurately, morphological operations based on chamfer metrics give a good approximation to morphological operations using Euclidean discs as structuring elements. First a formal definition of chamfer metrics is presented and some properties are discussed. Then, a number of morphological operations based on chamfer metrics are defined. These are the medial axis, the medial line, size distributions, anti-size distributions and the opening transform, A theoretical analysis of some properties of these operators is provided. This analysis concentrates on the relation between distance transformations and reconstructions, and the morphological operators just mentioned. This leads to a number of efficient algorithms for the computation of the morphological operators mentioned before. All algorithms (except for the opening transform) require a fixed number of image scans and are based on local operations only. An algorithm for the opening transform is presented which is 50 to 100 times faster than the brute force algorithm.
\end{abstract}

1991 Mathematics Subject Classification: 68U10, 51K99

Keywords \& Phrases: mathematical morphology, chamfer metrics, algorithms.

Note: This research was supported by the Foundation for Computer Science in the Netherlands (SION) with financial support from the Netherlands Organisation for Scientific Research (NWO). This research was part of a project in which the TNO Institute for Perception (Kampweg 5 , 3769 DE Soesterberg, The Netherlands), the Centre for Mathematics and Computer Science (Kruislaan 413, 1098 SJ Amsterdam, The Netherlands) and the Faculty of Mathematics and Computer Science of the University of Amsterdam (Kruislaan 403, 1098 SJ Amsterdam, The Netherlands) cooperate.

\section{Introduction}

This paper treats the relation between mathematical morphology $[9,16]$ and chamfer metrics [3]. It contains the analysis of properties of chamfer metrics and derives a description of morphological operators in terms of metrics and distance transforms. From this description, a number of algorithms for morphological operators is derived. This paper is restricted to morphology for binary images.

The definition of morphological operators requires the choice of a structuring element, a small set which is used as a probe. In many cases, mathematical morphology uses families of operators, which are constructed by applying a simple operation, such as the opening, with structuring elements of increasing size. In the continuous case, where images are subsets of the Euclidean plane, discs of increasing size are an appropriate choice for a family of structuring elements.

Report BS-R9309

ISSN 0924-0659

CWI

P.O. Box 4079, 1009 AB Amsterdam, The Netherlands 
In practical situations, image processing systems perform their operations on images defined on a discrete square grid. A common choice for a family of structuring elements on the discrete grid is a family of the form $n B=B \oplus \ldots \oplus B$, where $B$ is a square or a diamond. The disadvantage of this choice is the fact that such a family of structuring elements is quite dissimilar from the family of Euclidean discs. As chamfer metrics are a good approximation of the Euclidean metric, spheres in the chamfer metric seem to be a suitable choice as a family of structuring elements. Such a choice however poses some other problems, for example caused by the fact that larger spheres are in general not invariant under an opening by smaller spheres.

The goal of this paper is the integration of chamfer metrics into mathematical morphology. Both the theoretical and the practical aspect of such integration are discussed. The theoretical part consists of the construction of a number of morphological operators based on chamfer metrics and the analysis of such operators in terms of the metric. From this analysis, a number of efficient algorithms is derived, providing the practical part of the integration.

The paper starts with the description of some properties of general discrete metrics, a formal definition of the chamfer metric and a discussion of some of its properties. Then a number of morphological transformations, such as size distributions, anti-size distributions, the medial axis and the opening transform is defined using the chamfer metric and their properties are analysed. This analysis leads to a number of efficient algorithms for performing these operators.

Efficient algorithms for morphological operations have beed described by a number of authors. Groen and Foster [5] use lookup tables in order to speed up decisions based on inspection of the neighbourhood of a pixel. Vincent and Schmitt $[20,15]$ use queues in which only those pixels which must be processed are stored; pixels which need not to be processed are ignored. Van den Boomgaard and Van Balen [18] use a decomposition of the structuring element combined with a bitmapped storage structure for the image in order to construct efficient algorithms. The algorithms described by these authors are not applicable to operations based on the chamfer metric.

The chamfer metric has also been used for the construction of skeletons of objects $[4,11,13]$. (Here, the phrase "skeleton of an object" refers to a thin set with the same homotopy as the object. In section 4, the different ways in which the terms skeleton and medial axis are used in literature, will be discussed.) Authors using the chamfer metric for constructing skeletons usually compute skeletons by detecting ridges in the distance transform of an object or by a thinning algorithm in which pixels are scanned in order of increasing distance transform value. Another approach of skeletonisation is oriented more towards mathematical morphology and defines a skeleton as the locus of centers of maximal spheres in an object [16]. In this paper, the detection of centers of maximal spheres and thinning will be combined.

The organisation of the rest of this paper is as follows. In the next section, discrete metrics are introduced and some other definitions are presented. The third section presents chamfer metrics and some of their properties. This section also discusses algorithms for distance transforms and reconstructions for the chamfer metric, which are the building blocks for the algorithms to be presented in sections 4 through 6 . Section 4 presents the medial axis and the construction of a homotopy preserving medial line from the medial axis. Section 5 presents size distributions and

anti-size distributions. Section 6 presents the opening transform. The last section sums up the conclusions of this paper.

\section{Discrete Metrics}

In this section, discrete metrics are defined and some of their properties are described.

A metric on a set $E$ is a function $d: E \times E \rightarrow[0, \infty]$ satisfying the following conditions:

(1) $d(x, y)=0 \Leftrightarrow x=y$ for all $x, y \in E$. 
(2) $d(x, y)=d(y, x)$ for all $x, y \in E$.

(3) $d(x, y)+d(y, z) \leq d(x, z)$ for all $x, y, z \in E$.

Note that in this paper, metrics are allowed to assume the value $\infty$. This is necessary because later on, metrics will be constructed for which the distance between two points is defined as the length of a shortest path between them. If there is no path between two points, the distance between these points will be $\infty$. For chamfer metrics, which are a special case of shortest path metrics, all distances will be finite.

Let $d$ be a metric on a set $E$. Let $D$ denote the set $\{d(x, y) \mid x, y \in E\}$; this set is called the range of the metric. For each $d \in D$ open and closed spheres can be defined.

1. Definition. Let $d$ be a metric on a set $E$ and let $D$ be its range. Let $r \in D$ and $x \in E$. The closed sphere with radius $r$ and center $x$ is the set

$$
\bar{B}(x, r)=\{y \in E \mid d(x, y) \leq r\}
$$

2. Definition. Let $d$ be a metric on a set $E$ and let $D$ be its range. Let $r \in D$ and $x \in E$. The open sphere with radius $r$ and center $x$ is the set

$$
B(x, r)=\{y \in E \mid d(x, y)<r\}
$$

The rest of this paper will be concerned only with metrics which have a discrete range. Such metrics will be called discrete metrics:

3. Definition. A metric $d$ on a set $E$ is called a discrete metric if its range $\{d(x, y) \mid x, y \in E\}$ has no limiting points.

Thus, for each value $r>0$ in the range $D$ of a discrete metric $d$, there is a value $s \in D$ which is the largest value in $D$ smaller than $r$ and for each $r \in D$ there is a value $t \in D$ which is the smallest value in $D$ larger than $r$. Therefore, for a discrete metric each closed sphere of radius $r$ is also an open sphere of some larger radius $s$, which is the smallest number in $D$ larger than $r$. Each open sphere of radius $r>0$ is also a closed sphere of some smaller radius $s$ which is the largest number in $D$ such that $s<r$. In the sequel, we will sometimes write $B(r)$ for $B((0,0), r)$ and $\bar{B}(r)$ for $\bar{B}((0,0), r)$.

If $X$ is a subset of a discrete metric space $E$, it is possible to calculate for each point the shortest distance to a point outside the set.

4. Definition. Let $d$ be a discrete metric on a set $E$ and let $D$ be its range. Let $X$ be a bounded subset of $E$. The external distance transform $\rho_{X}^{\text {ext }}$ of $X$ is the function $E \rightarrow D$ defined by

$$
\rho_{X}^{\mathrm{ext}}(x)=\min _{y \in X^{C}} d(x, y)
$$

Note that the value of $\rho_{X}^{\text {ext }}(x)=0$ for $x \notin X$. The definition implies $B\left(x, \rho_{X}^{\text {ext }}(x)\right) \subset X$ for all $x \in X$. Using the definition of a metric, it can be seen that $\rho_{X}^{\text {ext }}(x) \leq \rho_{X}^{\text {ext }}(y)+d(x, y)$, for all $x, y \in E$. For a function $f: E \rightarrow \mathbf{R}$, the support $\operatorname{Supp}(f)$ of $f$ is defined as the set $\{x \in E \mid f(x) \neq 0\}$. The support $\operatorname{Supp}\left(\rho_{X}^{\text {ext }}\right)$ of $\rho_{X}^{\text {ext }}$ is the set $X$ itself.

For each point $x$ in a bounded subset $X$ of a discrete metric space $E$, it is possible to determine the largest closed sphere centered at $x$ and is contained in $X$. This leads to the notion of the internal distance transform. 
5. Definition. Let $d$ be a discrete metric on a set $E$ and let $D$ be its range. Let $X$ be a bounded subset of $E$. The internal distance transform $\rho_{X}^{\text {int }}$ of $X$ is the function $X \rightarrow D$ defined by

$$
\rho_{X}^{\text {int }}(x)=\max \{r \in D \mid \bar{B}(x, r) \subset X\}
$$

The internal distance transform can be calculated from the external distance transform. The value of $\rho_{X}^{\text {int }}(x)$ for an $x \in X$ is the largest number $r \in D$ such that $r<\rho_{X}^{\text {ext }}(x)$. Note that such a number exists because $d$ is a discrete metric.

Another ingredient for the algorithms to be presented later is the reconstruction. A reconstruction is the computation of a set as a union of spheres, which can be performed if the centers and radii of these spheres are given.

6. Definition. Let $d$ be a discrete metric on a set $E$ and let $D$ be its range. Let $f: E \rightarrow D$ be a function of bounded support. The open sphere reconstruction $R(f)$ of $f$ is defined by

$$
R(f)=\bigcup_{x \in \operatorname{Supp}(f)} B(x, f(x)) .
$$

Thus, the reconstruction is a union of open discs: an open disc with center $x$ and radius $f(x)$ is inserted in the set for each $x \in E$ with $f(x)>0$. An equivalent definition for $R(f)$ is

$$
R(f)=\{x \in E \mid d(x, y)<f(y) \text { for some } y \in E\} .
$$

It is also possible to define a reconstruction using closed spheres.

7. Definition. Let $d$ be a discrete metric on a set $E$ and let $D$ be its range. Let $X$ be a bounded subset of $E$ and let $f$ be a function $X \rightarrow D$. The closed sphere reconstruction $\bar{R}(f)$ of $f$ is defined by

$$
\bar{R}(f)=\bigcup_{x \in X} \bar{B}(x, f(x)) .
$$

The closed sphere reconstruction builds a set as a union of closed spheres. An equivalent definition is:

$$
\bar{R}(f)=\{y \in E \mid d(x, y) \leq f(x) \text { for some } x \in X\} .
$$

If $d$ is a metric on a set $E$, a point $y \in E$ is said to lie between two points $x, z \in E$ if $d(x, y)+d(y, z)=d(x, z)$. If $d$ is the Euclidean metric, $y$ lies between $x$ and $z$ if $y$ lies on the line segment from $x$ to $z$, which is the intuitive meaning of 'between'. Later we will need metrics for which, given two points $x$ and $y$ and a value $r$ in the range of $d$, a third point $z$ can be found at a distance $r$ from $x$, such that $y$ lies between $x$ and $z$.

8. Definition. Let $d$ be a metric on a set $E$ and let $D$ be its range. The metric $d$ is called extending if, for each $x, y \in E$ and $r \in E$, there is a $z \in E$ such that $d(x, y)+d(y, z)=d(x, z)$ and $d(y, z)=r$.

\section{Chamfer Metrics.}

The chamfer metric was introduced by Borgefors [3]. Her goal was the construction of a metric on the square grid which is a good approximation of the Euclidean metric and allows efficient computation. The chamfer metric is a metric defined on the square grid $\{p(1,0)+q(0,1) \mid p, q \in \mathbf{Z}\}$ or on the hexagonal grid $\{p(1,0)+q(1 / 2,1 / 2 \sqrt{3}) \mid p, q \in \mathbf{Z}\}$. These grids are illustrated in figure 1 . 
They are invariant under rotations and reflections. The group of symmetries of the square grid (leaving the origin fixed) contains four rotations, including the identity, and four line reflections. The symmetry group of the hexagonal grid contains six rotations, including the identity, and six reflections. Both the square grid and the hexagonal grid are representations of $\mathbf{Z}^{2}$. The only difference lies in the different symmetries of the two types of grids.

A metric on $\mathbf{Z}^{2}$ can be defined in the following way. Let $V=\left(v_{1}, \ldots, v_{k}\right)$ be a set of vectors in $\mathbf{Z}^{2}$ such that $v \in V$ implies $-v \in V$. These vectors are called prime vectors. Let $l$ be a function $V \rightarrow \mathbf{N}$ such that $l(v)=l(-v)$ and $l(v)>0$ for all $v$. The numbers $l(v)$ are called the weights of the prime vectors. It is also possible to use real numbers as weights, but that is not done in this paper. This is not a real restriction: if rational weights are to be used, they can be multiplied by a suitable scaling factor, yielding integer values. In practice, operations will be performed on a bounded grid and real numbers can be approximated with sufficient accuracy by rational numbers.

If $x$ and $y$ are two points in $\mathbf{Z}^{2}$, a path from $x$ to $y$ is defined as a sequence of points $x=$ $p_{0}, p_{1}, \ldots, p_{k}=y$ such that $p_{i}-p_{i-1} \in V$ for $i=1 \ldots k$. The length of this path is defined as :

$$
L\left(p_{0}, \ldots, p_{k}\right)=\sum_{i=1}^{k} l\left(p_{i}-p_{i-1}\right) .
$$

If $x=y$, we allow $p_{0}=x=y$ as a path from $x$ to $y$. The length of this path is 0 .

Let $x=p_{0}, \ldots, p_{k}=y$ be a path from $x$ to $y$. The differences $u_{i}=p_{i}-p_{i-1}$ are called the steps in the path. Any permutation $\sigma$ of the steps $u_{i}$ yields another path $p_{0}^{\prime}, \ldots, p_{k}^{\prime}$ from $x$ to $y$ given by $p_{i}^{\prime}=p_{i-1}^{\prime}+\sigma\left(u_{i}\right)$. Note that permutations do not affect the length of the path. Any sequence $u_{1}, \ldots, u_{k}$ of prime vectors such that $\sum_{i=1}^{n} u_{i}=y-x$ defines a path from $x$ to $y$ : take $p_{j}=x+\sum_{i=1}^{j} u_{j}$.

1. Theorem. With $\left(v_{1}, \ldots, v_{n}\right)$ and $l, L$ as defined above, a metric $d: \mathbf{Z}^{2} \times \mathbf{Z}^{2} \rightarrow[0, \infty]$ is defined by

$$
d(x, y)=\min \left\{L\left(p_{0}, \ldots, p_{k}\right) \mid k \in \mathbb{N} ; p_{0}, \ldots, p_{k} \text { is a path from } x \text { to } y\right\} .
$$

The proof of this theorem is simple: the first two properties of a metric are satisfied by construction and the third property can be verified using concatenation of paths. Note that $d(x, y)=\infty$ if there is no path between $x$ and $y$.

The construction described above defines a metric, but the chamfer metrics to be investigated in this paper will have some additional properties. Before these properties can be formulated, some definitions have to be made.

2. Definition. Let $\left\{v_{1}, \ldots, v_{n}\right\}$ be a set of prime vectors and let $l$ be a weight function. Then the normalised prime vectors $\hat{v}_{1}, \ldots, \hat{v}_{n}$ are defined by

$$
\hat{v}_{i}=v_{i} / l\left(v_{i}\right)
$$

Note that the normalised prime vectors are in general not points on the grid. Let $\mathbf{R}^{+}$denote the set of nonnegative reals.

3. Definition. Let $u$ and $w$ be two independent vectors in $\mathbf{R}^{2}$. The wedge between these two vectors is the set

$$
W_{u, w}=\left\{\lambda_{1} u+\lambda_{2} w \mid \lambda_{1}, \lambda_{2} \in \mathbf{R}^{+}\right\} .
$$

The wedge between two vectors is indeed a wedge shaped set, bounded by two half lines in the directions of the vectors. 
4. Definition. Let $V=\left\{v_{1}, \ldots, v_{n}\right\}$ be a set of prime vectors. Two independent vectors $u, w \in V$ are adjacent if $W_{u, w}$ contains no prime vectors other than $u$ and $w$.

An adjacency relation is defined for normalised prime vectors in the same way as for prime vectors. Note that $\hat{u}$ and $\hat{v}$ are adjacent if and only if $u$ and $v$ are adjacent.

5. Lemma. Let $V$ be a finite set of vectors in $\mathbf{Z}^{2}$ such that $V$ contains a pair of independent vectors and suppose that for each $v \in V,-v \in V$ as well, and that $-v$ is the only vector in $V$ which is collinear with $v$. Then for each $x \in \mathbf{Z}^{2}$ there are two adjacent vectors $u$ and $w$ in $V$ such that $x \in W_{u, w}$.

Proof. The lemma can be proving by ordering the prime vectors according to their angle with the positive $x$-axis. Under the given conditions, no two prime vectors have the same direction and two prime vectors are adjacent in the sense of lemma 4 if they are adjacent in the ordering according to their direction. Under the given conditions it can easily be seen that the plane is covered by wedges spanned by pairs of adjacent prime vectors.

Two different wedges intersect only in the origin, or they have a bounding half line in common.

If $u=\left(u_{x}, u_{y}\right)$ and $w=\left(w_{x}, w_{y}\right)$ are two points in $\mathbf{Z}^{2}$, the determinant $\operatorname{det}(u, w)$ is defined by $\operatorname{det}(u, w)=u_{x} w_{y}-u_{y} w_{x}$. Note that $u$ and $w$ are linearly dependent if and only if $\operatorname{det}(u, w)=0$.

Although a set of weighted prime vectors can define a metric as in theorem 1 , the metrics referred to in the literature as chamfer metrics form a subclass of such metrics. The next theorem gives a formal definition of this subclass; the consequences of this definition are described in the following lemmas.

6. Definition. A metric as defined above is called a chamfer metric if the following properties hold:

(1) The set $V$ of prime vectors contains a pair of linearly independent vectors.

(2) If $v \in V$, the only other vector in $V$ which is collinear with $v$ is $-v$.

(3) If $u$ and $w$ are two adjacent prime vectors, then $\operatorname{det}(u, w)= \pm 1$.

(4) The normalised prime vectors $\hat{v}_{1}, \ldots, \hat{v}_{n}$ lie on the boundary of a convex polygon.

Condition (3) in definition 6 implies that each grid point which lies in a wedge can be written as an integer combination of the prime vectors generating the wedge:

7. Lemma. Let $u$ and $w$ be two adjacent prime vectors and let $p=\lambda u+\mu w \in \mathbf{Z}^{2}$ for some $\lambda, \mu \in \mathbb{R}^{+}$. Then $\operatorname{det}(u, w)= \pm 1$ implies $\lambda$ and $\mu$ are integer.

Proof. From the equality

$$
\left(\begin{array}{ll}
u_{x} & w_{x} \\
u_{y} & w_{y}
\end{array}\right)\left(\begin{array}{l}
\lambda \\
\mu
\end{array}\right)=\left(\begin{array}{l}
p_{x} \\
p_{y}
\end{array}\right)
$$

it follows immediately that

$$
\left(\begin{array}{c}
\lambda \\
\mu
\end{array}\right)=\frac{1}{\operatorname{det}(u, w)}\left(\begin{array}{c}
w_{y} p_{x}-w_{x} p_{y} \\
u_{y} p_{x}-u_{x} p_{y}
\end{array}\right)
$$

Both $w_{y} p_{x}-w_{x} p_{y}$ and $u_{y} p_{x}-u_{x} p_{y}$ are integer, so $\lambda$ and $\mu$ are integer if $\operatorname{det}(u, w)= \pm 1$.

As there is a pair of independent prime vectors, it follows from lemma 5 that each grid point lies in some wedge. Lemma 7 implies that each grid point in a wedge can be written as an integer combination of the two prime vectors generating this wedge. This also immediately produces a path from 0 to each point in which only two prime vectors occur as steps. Because of translation invariance, it follows that for each $x, y \in \mathbf{Z}^{2}$, there is a path from $x$ to $y$ that contains at most two different prime vectors as steps. From property (4) in definition 6 it follows that such a path is a shortest path: 
8. Lemma. Suppose $p=m_{1} u_{1}+m_{2} u_{2}$ where $u_{1}$ and $u_{2}$ are adjacent prime vectors and $m_{1}, m_{2} \in$ N. Then $d(0, p)=m_{1} l\left(u_{1}\right)+m_{2} l\left(u_{2}\right)$.

Proof. It is clear that a path $P$ of length $m_{1} l\left(u_{1}\right)+m_{2} l\left(u_{2}\right)$ from 0 to $p$ exists. Now let $Q$ be a path from 0 to $p$ which contains each prime vector $v_{i} n_{i}$ times. Then the length of $Q$ is $\sum_{i=1}^{n} n_{i} l\left(v_{i}\right)$. We show that $L(P) \leq L(Q)$. We have

$$
\begin{aligned}
p & =\sum_{i=1}^{n} n_{i} v_{i}=\left(\sum_{j=1}^{n} n_{j} l\left(v_{j}\right)\right) \sum_{i=1}^{n}\left(\frac{n_{i} l\left(v_{i}\right)}{\sum_{j=1}^{n} n_{j} l\left(v_{j}\right)}\right) \hat{v}_{i} \\
& =L(Q) \sum_{i=1}^{n}\left(\frac{n_{i} l\left(v_{i}\right)}{\sum_{j=1}^{n} n_{j} l\left(v_{j}\right)}\right) \hat{v}_{i}
\end{aligned}
$$

and

$$
\begin{aligned}
p & =m_{1} u_{1}+m_{2} u_{2}=\left(m_{1} l\left(u_{1}\right)+m_{2} l\left(u_{2}\right)\right)\left\{\frac{m_{1} l\left(u_{1}\right)}{m_{1} l\left(u_{1}\right)+m_{2} l\left(u_{2}\right)} \hat{u}_{1}+\frac{m_{2} l\left(u_{2}\right)}{m_{1} l\left(u_{1}\right)+m_{2} l\left(u_{2}\right)} \hat{u}_{2}\right\} \\
& =L(P)\left\{\frac{m_{1} l\left(u_{1}\right)}{m_{1} l\left(u_{1}\right)+m_{2} l\left(u_{2}\right)} \hat{u}_{1}+\frac{m_{2} l\left(u_{2}\right)}{m_{1} l\left(u_{1}\right)+m_{2} l\left(u_{2}\right)} \hat{u}_{2}\right\}
\end{aligned}
$$

These are two ways of writing $p$ as a multiple of a convex combination of normalised prime vectors. Therefore

$$
L(Q) \sum_{i=1}^{n}\left(\frac{n_{i} l\left(v_{i}\right)}{\sum_{j=1}^{n} n_{j} l\left(v_{j}\right)}\right) \hat{v}_{i}=L(P)\left\{\frac{m_{1} l\left(u_{1}\right)}{m_{1} l\left(u_{1}\right)+m_{2} l\left(u_{2}\right)} \hat{u_{1}}+\frac{m_{2} l\left(u_{2}\right)}{m_{1} l\left(u_{1}\right)+m_{2} l\left(u_{2}\right)} \hat{u_{2}}\right\} .
$$

Therefore, the two convex combinations of normalised unit vectors point in the same direction. The convex combination $\sum_{i=1}^{n}\left(\frac{n_{i} l\left(v_{i}\right)}{\sum_{j=1}^{n} n_{j} l\left(v_{j}\right)}\right) \hat{v}_{i}$ lies in the polygon. As $\hat{u}_{1}$ and $\hat{u}_{2}$ are adjacent vectors and lie on the boundary of a convex polygon, a convex combination of $\hat{u}_{1}$ and $\hat{u}_{2}$ lies on the boundary of the polygon. Therefore, the length of $\frac{m_{1} l\left(u_{1}\right)}{m_{1} l\left(u_{1}\right)+m_{2} l\left(u_{2}\right)} \hat{u}_{1}+\frac{m_{2} l\left(u_{2}\right)}{m_{1} l\left(u_{1}\right)+m_{2} l\left(u_{2}\right)} \hat{u}_{2}$ is at least as large as the length of $\sum_{i=1}^{n}\left(\frac{n_{i} l\left(v_{i}\right)}{\sum_{j=1}^{n} n_{j} l\left(v_{j}\right)}\right) \hat{v}_{i}$. Consequently, $L(Q)$ is at least as large as $L(P)$.

There are many different sets of prime vectors and weights which generate the same metric. If $u$ and $w$ are adjacent prime vectors, the addition of $u+w$ and $-u-w$ with weight $l(u)+l(w)$ to the set of prime vectors produces the same chamfer metric as the one generated by the original set of prime vectors. The number of shortest paths between two points is however increased by enlarging the set of prime vectors, because two steps $u$ and $w$ in a path can be replaced by a single step $u+w$. There is, however, the following theorem:

9. Theorem. Suppose $V$ is a set of prime vectors provided with weights, generating a chamfer metric such that the normalised prime vectors are the corners of a convex polygon. Suppose $p=m_{1} u_{1}+m_{2} u_{2}$, where $u_{1}$ and $u_{2}$ are two adjacent prime vectors and $m_{1}, m_{2} \in \mathbf{N}$. Then a shortest path from 0 to $p$ contains only steps $u_{1}$ and $u_{2}$.

Proof. Let $P$ and $Q$ be paths as described in the proof of the previous theorem. It must be shown that the only $n_{i}$ which have a nonzero value are those corresponding to $u_{1}$ and $u_{2}$. The line segment between the normalised prime vectors $\hat{u}_{1}$ and $\hat{u}_{2}$ is a edge of the convex hull of the 
set of normalised prime vectors and they are the only normalised prime vectors on this edge of the polygon. If $L(P)=L(Q)$, it follows that

$$
\sum_{i=1}^{n}\left(\frac{n_{i} l\left(v_{i}\right)}{\sum_{j=1}^{n} n_{j} l\left(v_{j}\right)}\right) \hat{v}_{i}=\frac{m_{1} l\left(u_{1}\right)}{m_{1} l\left(u_{1}\right)+m_{2} l\left(u_{2}\right)} \hat{u}_{1}+\frac{m_{2} l\left(u_{2}\right)}{m_{1} l\left(u_{1}\right)+m_{2} l\left(u_{2}\right)} \hat{u}_{2} .
$$

As there is only one way of writing a point on a edge of a convex polygon as a convex combination of the corners of the polygon, it follows that the two convex combinations above are the same, and that the only nonzero $n_{i}$ 's are those corresponding to $u$ and $w$.

If not all normalised prime vectors are corners of a convex polygon, different types of shortest paths can occur. Suppose for example that $v_{x}=(1,0)$ and $v_{y}=(0,1)$ are prime vectors with weight 1 and $v_{x y}=(1,1)$ is a prime vector with weight 2 . Then $(0,0)-(1,1)$ is a shortest path from $(0,0)$ to $(1,1)$ containing a single step $v_{x y}$, but the path $(0,0)-(1,0)-(1,1)$ contains steps $v_{x}$ and $v_{y}$ and is a shortest path as well. If the weight of $v_{x y}$ is 3 , the path $(0,0)-(1,0)-(1,1)$ is even shorter than the path $(0,0)-(1,1)$.

Figure 1 shows the square grid and the hexagonal grid, each with an example set of primitives vectors. The primitive vectors divide the grid in wedges, which are also indicated.
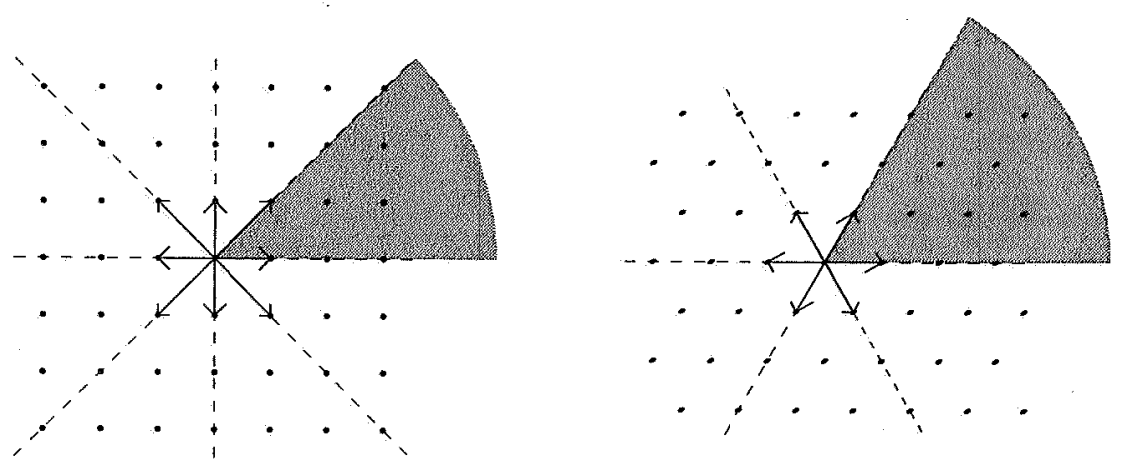

Figure 1. The square grid (a) and the hexagonal grid (b) with a number of primitive vectors. The primitive vectors divide the grid in a number of wedges. In each grid, one of these wedges has been shaded.

The definition of chamfer metrics can be extended to higher dimensions. For $\mathbf{Z}^{3}$, for example, the adjacency relation is defined for triples of vectors and the determinant is replaced by a $3 \times 3$ determinant.

Some often used chamfer metrics are represented in figure 2. Each square containing a number corresponds with a prime vector. The position of the square relative to the center square is the prime vector and the number in the square is the weight of this prime vector. For the 5-7-metric, for example, there are eight prime vectors. The prime vectors $(1,0),(0,1),(-1,0)$ and $(0,-1)$ have weight 5 ; the prime vectors $(1,1),(-1,1),(-1,-1)$ and $(1,-1)$ have weight 7 .

Verwer [19] has analysed the accuracy of chamfer metrics on a square grid as an approximation to the Euclidean metric. The city block metric and the chess board metric are accurate within 17.16\%. The 5-7-metric is accurate within $4.21 \%$ and the $5-7-11$-metric is accurate within $1.79 \%$.

The examples in the rest of this paper will discuss the 5-7-metric, although similar results can be obtained for other metrics. The reasons for this choice are the following. The city block metric and the chess board metric are not good approximations of the Euclidean metric. The chamfer 

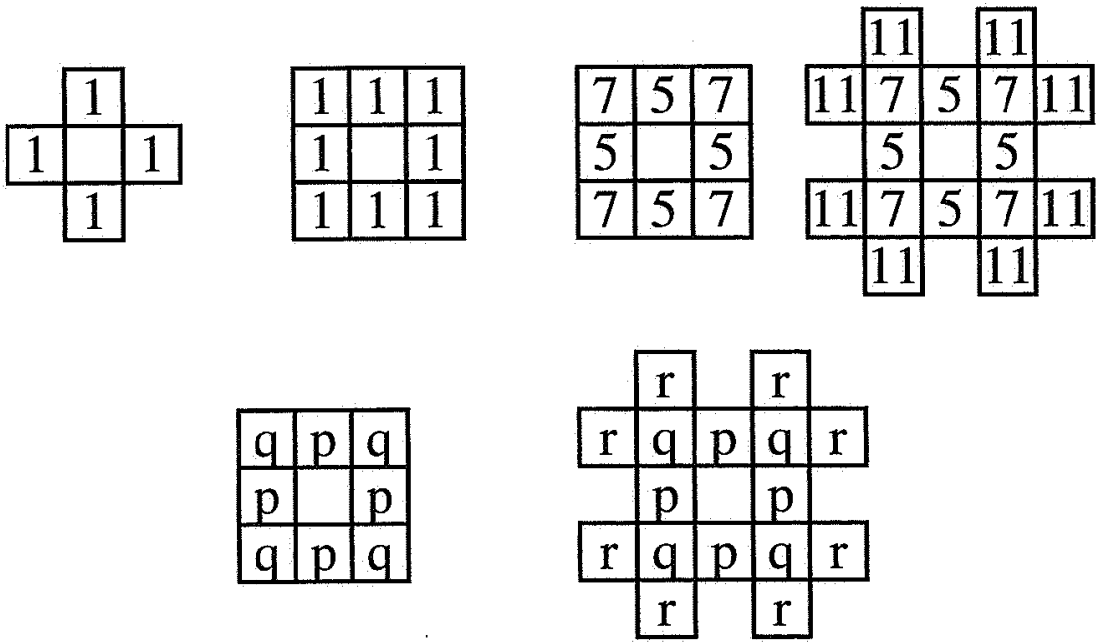

Figure 2: The masks describing chamfer metrics. The top line shows the masks for the city block metric, the chess board metric, the 5-7-metric and the 5-7-11-metric, respectively. Metrics defined by choosing other weights are referred to as p-q-metrics or $p$-q-r-metrics.

metrics on the hexagonal grid have the disadvantage that there are no imaging devices producing images on a hexagonal grid. Both the 5-7-metric and the 5-7-11-metric are good approximations of the Euclidean metric. In this paper, the 5-7-metric is used, because the 5-7-11-metric is not extending; therefore the algorithm presented in section 5 for computing the medial axis cannot handle the 5-7-11-metric. This restriction is not too problematic, because the gain in accuracy from the 5-7-metric to the 5-7-11-metric is small compared with the gain in accuracy from the city block or chess board metric to the 5-7-metric.

As the distance between two points in a chamfer metric is always an integer, every chamfer metric is a discrete metric. If the weights of adjacent prime vectors in the definition of a chamfer metric have greatest common divisor (gcd) 1 , something more can be said. In that case, the range of the metric is the set of all but a finite number of natural numbers.

10. Theorem. Let $D$ be a chamfer metric and let $D$ be its range. Let $l_{1}$ and $l_{2}$ be the weights of two adjacent prime vectors such that $\operatorname{gcd}\left(l_{1}, l_{2}\right)=1$. Then $\mathbf{N}-D$ is finite.

Proof. Suppose $l_{1}$ is the weight of $v_{1}$ and $l_{2}$ is the weight of $v_{2}$. For each $n_{1}, n_{2} \in \mathbf{N}$ the vector $x=n_{1} v_{1}+n_{2} v_{2} \in W_{v_{1}, v_{2}}$ satisfies $d(0, x)=n_{1} l_{1}+n_{2} l_{2}$. Therefore, $D$ contains every number which can be written as $n_{1} l_{1}+n_{2} l_{2}$ with $n_{1}, n_{1} \in \mathbb{N}$. As $\operatorname{gcd}\left(l_{1}, l_{2}\right)=1$, there are integers $m_{1}$ and $m_{2}$ such that any integer $t$ can be written as an integer combination of $l_{1}$ and $l_{2}$ in exactly the following ways $[17]$ :

$$
t=\left(m_{1} t+l_{2} k\right) l_{1}+\left(m_{2} t-l_{1} k\right) l_{2} \quad(k \in \mathbf{Z}) .
$$

The possibilities using only nonnegative coefficients for $l_{1}$ and $l_{2}$ are found by solving the equations $m_{1} t+l_{2} k \geq 0$ and $m_{2} t-l_{1} k \geq 0$. These inequalities imply that $k$ must lie in the segment $\left[-\frac{m_{1}}{l_{2}} t, \frac{m_{2} t}{l_{1}} t\right]$. The length of this interval is $t / l_{1} l_{2}$, so if $t \geq l_{1} l_{2}$ The length of the interval is at least 1 and an integer value for $k$ can be found in the interval. Therefore, any integer $t \geq l_{1} l_{2}$ occurs as a distance value. Therefore, there are at most $l_{1} l_{2}$ natural numbers which are not in the range of the metric.

If it is known that all integers above a known bound are contained in the range, the range can be determined by checking the natural numbers below this bound. The range of the 5-7-metric, for example, is $\mathbf{N}-\{1,2,3,4,6,8,9,11,13,16,18,23\}$. This structure of the range of a chamfer metric 
enables the computation of the internal distance transform from the external distance transform. For each point, the internal distance transform value of a point with external distance transform value $r$ is the smallest $s \in D$ such that $s<r$. For $r$ larger that a certain bound, this value is $r-1$; for other values a lookup table can be constructed in order to facilitate easy computation.

Not all chamfer metrics are extending, but the p-q-metrics are. This can be seen from the fact that if $v_{1}$ and $v_{2}$ are two adjacent vectors, one of them has weight $p$ and the other one has weight $q$. Now let two points $x$ and $y$ be given, and let $r$ be a value in the range of the p-q-metric. We must find a point $z$ such that $d(x, y)+d(y, z)=d(x, z)$ and $d(y, z)=r$. The vector $y-x$ lies in a wedge $W_{v_{1}, v_{2}}$, spanned by two adjacent prime vectors $v_{1}$ and $v_{2}$. Without loss of generality it can be assumed that $v_{1}$ has weight $p$ and $v_{2}$ has weight $q$. It is possible to write $r=n_{1} p+n_{2} q$. Now let $z$ be the point $y+n_{1} v_{1}+n_{2} v_{2}$. Then $d(y, z)=n_{1} p+n_{2} q=r$ and $d(x, z)=d(x, y)+d(y, z)$ because a shortest path from $x$ to $z$ can be found by taking a shortest path from $x$ to $y$ and then proceeding to the points $y+v_{1}, y+2 v_{1}, \ldots, y+n_{1} v_{1}, y+n_{1} v_{1}+v_{2}, \ldots, y+n_{1} v_{1}+n_{2} v_{2}=z$.

Distance transforms and reconstructions can be computed efficiently for chamfer metrics. Both can be computed in two image scans: a forward scan, in which pixels are scanned from top to bottom and from left to right, and a backward scan, in which pixels are scanned from bottom to top and from right to left. In the forward scan, pixels values are updated using information from so-called backward neighbours; in the backward scan, pixel values are updated using information from forward neighbourhoods.
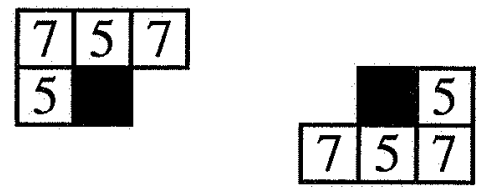

Figure 3: The backward and forward masks for the 5-7-metric.

The forward and backward masks associated with the 5-7-metric are shown in figure 3. This figure should be interpreted as follows: if $\left(x_{1}, x_{2}\right)$ are the coordinates of a pixel, its backwards neighbours are $n_{1}=\left(x_{1}, x_{2}-1\right)$ and $n_{3}=\left(x_{1}-1, x_{2}\right)$ at distance $d_{1}=d_{3}=5$ and $n_{2}=\left(x_{1}-1, x_{2}-1\right)$ and $n_{4}=\left(x_{1}+1, x_{1}-1\right)$ at distance $d_{2}=d_{4}=7$. The forward neighbourhood mask should be interpreted in the same way.

The algorithms for the distance transformation and the reconstruction are well known from the literature [3] and are described here only for the sake of completeness. They use a rectangular grid where the pixels are denoted by $x_{i}$ and their values by $v_{i}$.

11. Algorithm. The external distance transform of an object $X$ with respect to a chamfer metric.

(1) Initialize: $v_{i}:=\infty$ if $x_{i} \in X, v_{i}:=0$ otherwise.

(2) For all pixels $x_{i}$ in forward scanning order: if $\min \left\{v_{j}+d_{j} \mid x_{j}\right.$ backward neighbour of $\left.x_{i}\right\}<v_{i}$, then replace $v_{i}$ by this value.

(3) For all pixels $x_{i}$ in backward scanning order: if $\min \left\{v_{j}+d_{j} \mid x_{j}\right.$ forward neighbour of $\left.x_{i}\right\}<v_{i}$, then replace $v_{i}$ by this value.

12. Algorithm. The computation of the internal distance transform of a bounded set $X$ for a chamfer metric with range $D$.

(1) Compute the external distance transform of $X$.

(2) For each $x_{i} \in X$ : replace $v_{i}$ by $\max \left\{r \in D \mid r<v_{i}\right\}$. 
13. Algorithm. The open sphere reconstruction of a function $f$ with respect to a chamfer metric.

(1) Initialise: $v_{i}:=f\left(x_{i}\right)$.

(2) For all pixels $x_{i}$ in forward scanning order: if $\max \left\{v_{j}-d_{j} \mid x_{j}\right.$ backward neighbour of $\left.x_{i}\right\}>v_{i}$, replace $v_{i}$ by this value.

(3) For all pixels $x_{i}$ in backward scanning order: if $\max \left\{v_{j}-d_{j} \mid x_{j}\right.$ forward neighbour of $\left.x_{i}\right\}>v_{i}$, replace $v_{i}$ by this value.

(4) Determine the set of pixels $x_{i}$ with $v_{i}>0$.

14. Algorithm. The closed sphere reconstruction of a function $f$ on support $X$ with respect to a chamfer metric.

(1) Initialise: $v_{i}=f\left(x_{i}\right)$ if $x_{i} \in X ; v_{i}:=-\infty$ otherwise.

(2) For all pixels $x_{i}$ in forward scanning order: if $\max \left\{v_{j}-d_{j} \mid x_{j}\right.$ backward neighbour of $\left.x_{i}\right\}>v_{i}$, replace $v_{i}$ by this value.

(3) For all pixels $x_{i}$ in backward scanning order: if $\max \left\{v_{j}-d_{j} \mid x_{j}\right.$ forward neighbour of $\left.x_{i}\right\}>v_{i}$, replace $v_{i}$ by this value.

(4) Determine the set of pixels $x_{i}$ with $v_{i} \geq 0$.

\section{The Medial Axis and the Medial Line}

The literature shows great confusion concerning the terms 'medial axis' and 'skeleton'. There are two types of sets which are denoted by these terms. One is the locus of centers of maximal spheres in an object, the other one is a thin subset of an object which lies in the middle of the object and has the same homotopy as the object. In the continuous case, this does not lead to great difficulties, because usually, the differences between these two sets are very small [10].

In the discrete case, this confusion is more serious, because the locus of centers of maximal spheres often is a set with much more connected components than the object itself. In general, it can be said that authors from the field of mathematical morphology $[8,16]$ use the term skeleton for the locus of centers of maximal spheres, while others $[2,4,13]$ call this set the medial axis and use the term skeleton for a thin set having the same homotopy as the object. There are, however, exceptions to this rule [11]. Other names occurring in the literature for the different types of sets are medial line, symmetric axis and homotopic thinning.

In this paper, the term medial axis will denote the locus of centers of maximal spheres and the term medial line will denote a thin set of the same homotopy as the object. In this section, an algorithm is presented which calculates the medial axis defined by families of spheres corresponding with several chamfer metrics.

1. Definition. Let $d$ be a discrete metric on a set $E$. Let $X$ be a bounded subset of $E$. A sphere $\bar{B}(x, r) \subseteq X$ is called a maximal sphere if, for each sphere $\bar{B}(y, s)$, the inclusion $\bar{B}(x, r) \subseteq \bar{B}(y, s) \subseteq$ $X$ implies $\bar{B}(x, r)=\bar{B}(y, s)$.

2. Definition. Let $d$ be a discrete metric on a set $E$ and let $X$ be a bounded subset of $E$. The medial axis $M_{X}$ of $X$ is the set of centers of maximal spheres in $X$.

If the medial axis of an object is given, together with the value of the external distance transform in the medial axis points, the object can be reconstructed using the equality

$$
X=\bigcup_{s \in M_{X}}\left\{x \in \mathbf{Z}^{2} \mid d(x, s)<\rho_{X}^{\text {ext }}(s)\right\}
$$


The restriction of the distance transform to the medial axis is sometimes called the quench function [16]. The reconstruction property can be seen as follows: for each $x \in X$, the set of spheres $B(y, s)$ containing $x$ and contained in $X$ is not empty because $\bar{B}(x, 0)=\{x\}$, which is also an open sphere of some radius ( $d$ is discrete), is such a sphere. The set of all such spheres is partially ordered by inclusion. Because $X$ is bounded, it has a finite number of elements. Hence, there is a maximal ball $B(y, R)$ containing $x$ where $y$ is included in the medial axis. Therefore, $x \in\left\{z \in \mathbf{Z}^{2} \mid d(z, y)<\right.$ $\left.\rho_{X}^{\text {ext }}(y)\right\} \subseteq \bigcup_{s \in M_{X}}\left\{z \in \mathbf{Z}^{2} \mid d(z, s)<\rho_{X}^{\text {ext }}(s)\right\}$.

The inclusion in the other direction can easily be verified using the definition of the distance transform.

For a general metric, it can be difficult to compute the medial axis, but for extending metrics we can use the following result:

3. Theorem. Let $d$ be an extending metric on a set $E$. Let $X$ be a bounded subset of $E$. A point $x \in X$ is the center of a maximal sphere if and only if there is no $y \neq x$ such that $\rho_{X}^{\text {int }}(y) \geq \rho_{X}^{\text {int }}(x)+d(x, y)$ (" $x$ has no upstream").

Proof. 'only if': Suppose that there exists an $y \neq x$ such that $\rho_{X}^{\text {int }}(y) \geq \rho_{X}^{\text {int }}(x)+d(x, y)$. In that case every $z \in \bar{B}\left(x, \rho_{X}^{\text {int }}(x)\right)$ satisfies

$$
d(z, y) \leq d(z, x)+d(x, y) \leq \rho_{X}^{\text {int }}(x)+d(x, y) \leq \rho_{X}^{\text {int }}(y) .
$$

Therefore $\bar{B}\left(y, \rho_{X}^{\text {int }}(y)\right)$ is a sphere containing $\bar{B}\left(x, \rho_{X}^{\text {int }}(x)\right)$ and contained in $X$. Therefore $x$ is not the center of a maximal sphere.

'if': Suppose $x$ is not the center of a maximal sphere. Then the sphere with center $x$ and radius $\rho_{X}^{\text {int }}(x)$ must be contained in a closed sphere with center $y \neq x$ and radius $\rho_{X}^{\text {int }}(y)$. Let $z$ be a point such that $d(y, x)+d(x, z)=d(y, z)$ and $d(x, z)=\rho_{X}^{\text {int }}(x)$. Such a point exists because $d$ is extending. From $z \in \bar{B}\left(x, \rho_{X}^{\text {int }}(x)\right) \subset \bar{B}\left(y, \rho_{X}^{\text {int }}(y)\right)$ it follows that $d(z, y) \leq \rho_{X}^{\text {int }}(y)$.

From these relations it can be deduced that

$$
\rho_{X}^{\mathrm{int}}(y)-\rho_{X}^{\mathrm{int}}(x) \geq d(z, y)-\rho_{X}^{\mathrm{int}}(x)=d(z, y)-d(x, z)=d(x, y) .
$$

If this theorem is to be used for the computation of the medial axis of a set, it is necessary to check every pair of points in order to see if one point is the upstream of the other. In the case of the p-q-metric, however, the search can be limited using the following result:

4. Theorem. Let $X$ be a.bounded subset of $\mathbf{Z}^{2}$, provided with the p-q-metric, and let $x \in X$. If there is a point $y$ such that $\rho_{X}^{\text {int }}(y) \geq \rho_{X}^{\text {int }}(x)+d(x, y)$, there is also an 8-neighbour $z$ of $x$ satisfying $\rho_{X}^{\text {int }}(z) \geq \rho_{X}^{\text {int }}(x)+d(x, z)$.

Proof. Let $x$ and $y$ be points as described above. Then we have

$$
\bar{B}\left(x, \rho_{X}^{\mathrm{int}}(x)\right) \subseteq \bar{B}\left(y, \rho_{X}^{\mathrm{int}}(y) y\right) \subseteq X
$$

There exists an 8-connected shortest path (not necessarily unique) from $x$ to $y$. Let $z$ be the neighbour of $x$ in such a path. It is now sufficient to prove that

$$
\bar{B}\left(x, \rho_{X}^{\text {int }}(x)\right) \subseteq \bar{B}\left(z, \rho_{X}^{\text {int }}(x)+d(x, z)\right) \subseteq \bar{B}\left(y, \rho_{X}^{\text {int }}(y)\right) .
$$

The first inclusion follows from

$$
d(x, p) \leq \rho_{X}^{\mathrm{int}}(x) \Rightarrow d(z, p) \leq d(z, x)+d(x, p) \leq d(z, x)+\rho_{X}^{\mathrm{int}}(x) .
$$


The second inclusion follows from:

$$
\begin{gathered}
d(z, p) \leq \rho_{X}^{\text {int }}(x)+d(x, z) \Rightarrow \\
d(y, p) \leq d(y, z)+d(z, p) \leq d(x, z)+d(z, y)+\rho_{X}^{\text {int }}(x)=d(x, y)+\rho_{X}^{\text {int }}(x) \leq \rho_{X}^{\text {int }}(y) .
\end{gathered}
$$

Here we use $d(x, z)+d(z, y)=d(x, y)$, which is true because $z$ lies on a shortest path from $x$ to $y$.

The previous theorems suggest the following algorithm for the detection of the centers of maximal spheres of the $\mathrm{p}$-q-metric in an object $X$ :

5. Algorithm. The computation of the medial axis of a bounded subset $X$ of $Z^{2}$ with respect to the $p$-q-metric.

(1) Calculate the internal distance transformation of the object.

(2) Detect all points $x$ which have no 8 -neighbour $y$ satisfying $\rho_{X}^{\text {int }}(y) \geq \rho_{X}^{\text {int }}(x)+d(x, y)$.

This algorithm finds the centers of maximal spheres in 4 image scans. Arcelli and Sanniti di Baja [2] have obtained a similar result for the special case of the 3-4-metric. The algorithm presented here works for all extending metrics and has been implemented for the 5-7-metric. Figure 4 shows a binary image and its medial axis.

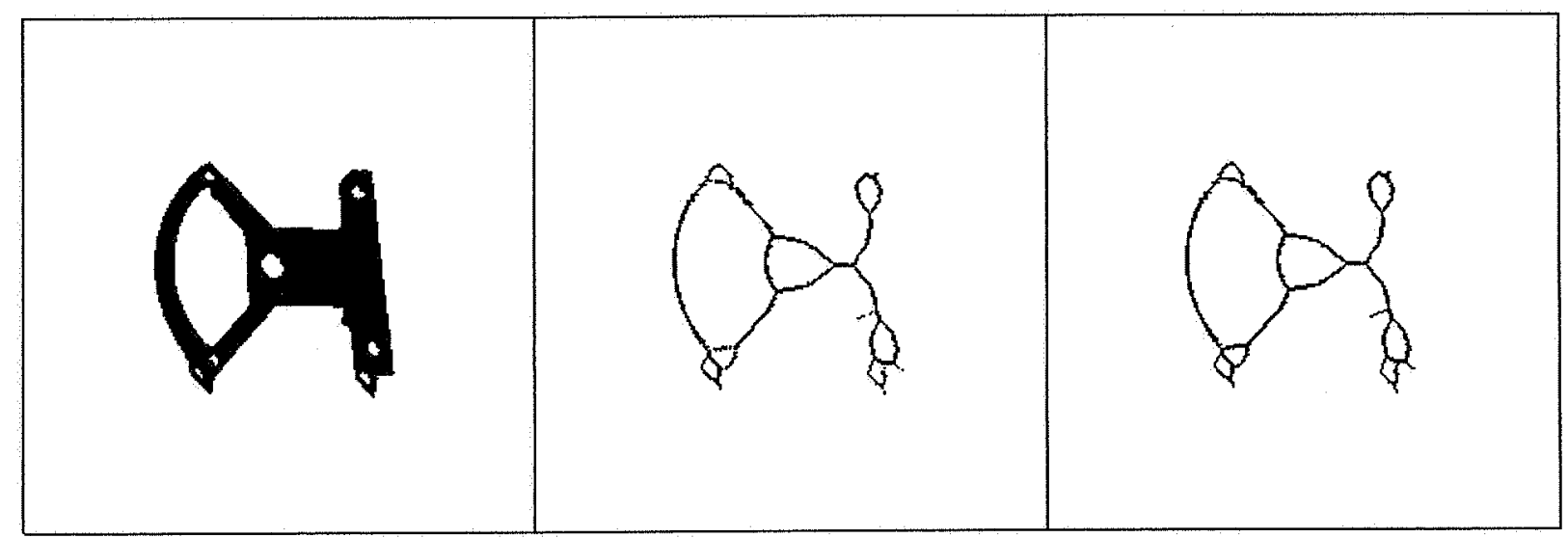

Figure 4. A binary object (a), its medial axis (b) and the medial line calculated by Hilditch anchor skeletonisation (c).

Note that the algorithm described above does not work in the case of the 5-7-11-metric. This can be seen in the following example:

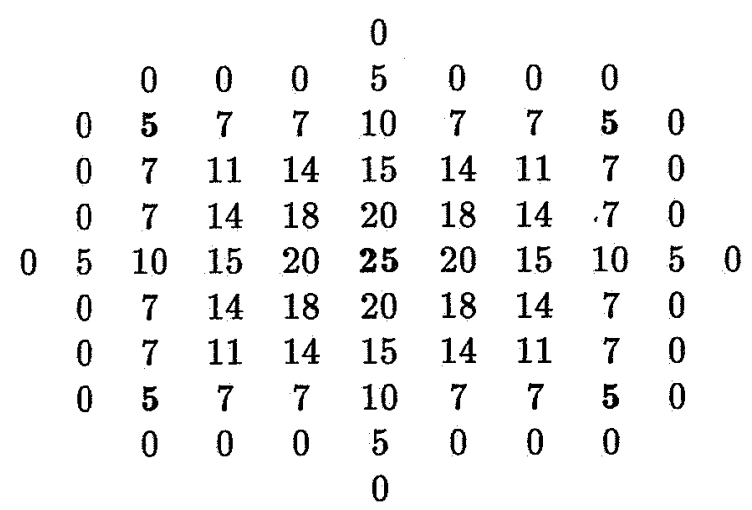


The picture represents an object $X$, which is a closed sphere of radius 25 . The numbers indicate the values of the internal distance transform. The only maximal sphere which occurs is the complete set $X$. Yet all points having a distance transform value marked in boldface satisfy the no-upstream condition, even not only neighbouring pairs are considered. The detection of the medial axis associated with the 5-7-11-metric is a subject of ongoing research.

In general, the medial axis of an object will have more connected components that the object itself. Therefore, it is interesting to look at medial lines. A medial line $S_{X}$ of a bounded subset $X$ of $\mathbf{Z}^{2}$ is a subset of $X$ which is thin, has the same homotopy as $X$ and lies in the middle of $X$. The medial line can be used as a description or a compact representation of shape. A medial line of a subset of $\mathbf{R}^{2}$ can be defined without too much trouble [10], but the definition of a medial line for discrete sets is much more intricate.

Many authors have worked on the problem of defining a medial line for discrete sets. One approach $[1,4,11,12,13]$ starts with choosing some "special configuration" points as a subset of the medial line. In general, this set will not have the correct homotopy. This is repaired by computing arcs between the points already selected in such a way that the resulting set has the correct homotopy. The difficult part is to prove that the resulting set has indeed the correct topology. Some authors provide complete correctness proofs $[11,12,13]$, some $[1,4]$ do not.

Another approach $[16,6]$ is based on thinning. All object pixels are scanned in some order, and they are removed if this can be done without changing the homotopy of the object. At the end, a set of pixels remains, none of which can be removed without changing the homotopy of the set. This set is a medial line.

We present a medial line which contains the medial axis. The knowledge of the medial line, together with the value of the distance transform on the points in the medial line suffices for the reconstruction of the object.

Our medial line algorithm is based on the work of Hilditch [6]. Her algorithm is based on thinning. In the present situation, thinning is performed under the condition that a medial axis point can never be removed, even if this removal would not change the homotopy. The resulting set is sometimes called an anchor skeleton. An example of a medial line of this type is shown in figure 4 .

The author conjectures that a homotopy-preserving medial line can also be derived from the medial axis using a path-climbing algorithm ${ }^{1}$, but has not been able to prove the correctness of his algorithm.

An example of the proposed medial line is shown in figure 4.

\section{Size Distributions and Anti-Size Distributions}

In this section, size distributions and anti-size distributions based on the chamfer metric are constructed and efficient algorithms for performing these operations are presented.

1. Definition. Let $E$ be some set and let $\Lambda$ be some totally ordered set. A size distribution $[8,16]$ is a family $\left\{\alpha_{r}\right\}_{r \in \Lambda}$ of operators mapping subsets of $E$ to subsets of $E$ such that for all $X, Y \subset E$, $r, s \in \Lambda$

\footnotetext{
1 This algorithm is based on the work of Dorst [4] and Niblack et al. [13]. It is a steepest path climbing type of algorithm. Our algorithm differs with those of Dorst and Niblack et al. in the choice of starting points for the paths: the starting points in our algorithm are the medial axis points and those points which form a one or two pixel wide connection between larger parts of the object. Although this algorithm seems to produce medial lines of the correct homotopy, the author has not been able to find a proof for this. The problem lies in necks of an object, which are narrow connections between two wider parts of the object. It must be shown that there are centers of maximal spheres in such a narrow part, and that paths of steepest ascent leave in both directions to the wider parts of the object.
} 
(1) $X \subset Y \Rightarrow \alpha_{r}(X) \subset \alpha_{r}(Y)$.

(2) $\alpha_{r}(X) \subset X$.

(3) $\alpha_{r} \circ \alpha_{r}=\alpha_{r}$.

(4) $\alpha_{r} \circ \alpha_{s}=\alpha_{\max (r, s)}$.

The first three conditions are exactly the conditions which are used to define that $\alpha_{r}$ is an opening [16]. The fourth condition describes the composition behaviour of these openings. Note that the third condition is a consequence of the fourth; it is written down in order to clarify the fact that each $\alpha_{r}$ is an opening.

In the sequel, the index set $\Lambda$ will be $D$. The goal is to define a size distribution based on spheres in a discrete metric. A first attempt could be to let each $\alpha_{r}$ be a structural opening [14] with the sphere $\bar{B}(r)$. This opening is defined by

$$
X \circ \bar{B}(r)=\bigcup_{\bar{B}(h, r) \subset X} \bar{B}(h, r) .
$$

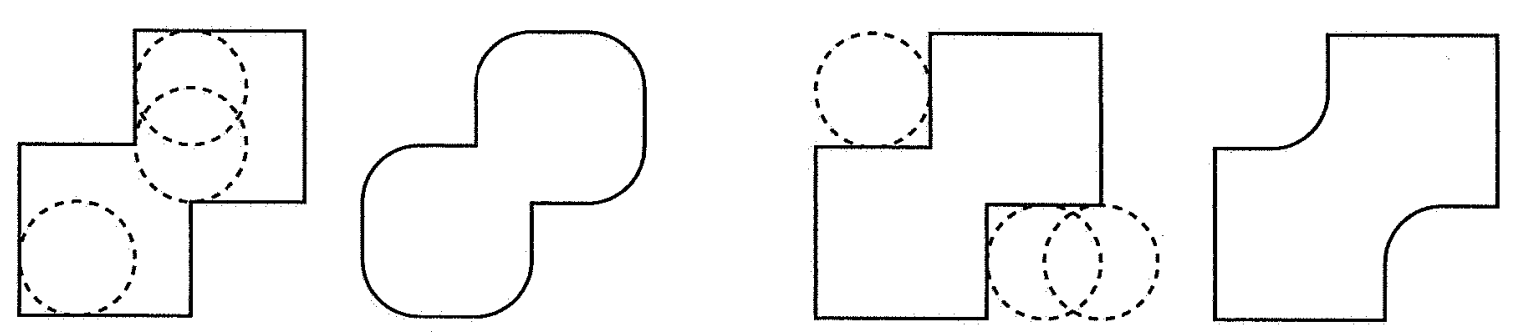

Figure 5: Examples of the structural opening (a) and closing (b) of a shape $X$ with a structuring element $B$. The opening is found by fitting the structuring element $B$ in the object $X$. The structural closing is found by fitting the structuring element $B$ in the background of $X$.

The structural opening is illustrated in figure 5. If $X$ is the image and $B$ is the structuring element, $X \circ B$ is defined as the union of all translates $B_{h}$ of $B$ which fit in $X$. This family of structural openings satisfies the first three conditions, but not the fourth one. This can be seen from a simple example using the 5-7-metric. Let $X=\bar{B}(7)$; as $\bar{B}(7) \circ \bar{B}(5)=\bar{B}(5)$ we get that

$$
(X \circ \bar{B}(7)) \circ \bar{B}(5)=\bar{B}(5) \neq X=X \circ \bar{B}(7) \text {. }
$$

However, it can be seen [9] that the following function family is indeed a size distribution:

$$
\alpha_{r}(X)=\bigcup_{s \geq r} X \circ \bar{B}(s)
$$

As each $\alpha_{r}$ is an opening, size distributions are not only useful in the analysis of sizes, but the individual operations can also be used as an alternative for the structural opening with a single sphere.

An algorithm for the calculation of $\alpha_{r}(X)$ is suggested by the following theorem:

2. Theorem. Let $d$ be a discrete metric on a set $E$ and let $D$ be its range. Let $X$ be a bounded subset of $E$ and $r \in D$. Then

$$
\begin{aligned}
\alpha_{r}(X) & =\bigcup_{\rho_{X}^{\text {ext }}(x)>r} B\left(x, \rho_{X}^{\text {ext }}(x)\right) \\
& =\bigcup_{\rho_{X}^{\text {int }}(x) \geq r} \bar{B}\left(x, \rho_{X}^{\text {int }}(x)\right)
\end{aligned}
$$


Proof. First suppose that $x \in \alpha_{r}(X)$. Then $x \in X \circ \bar{B}(s)$ for some $s \geq r$. Thus, there is a $y \in X$ such that $x \in \bar{B}(y, s) \subset X$. The second inclusion implies $\rho_{X}^{\text {ext }}(y)>s \geq r$, so $x \in \bar{B}(y, s) \subset$ $B\left(y, \rho_{X}^{\text {ext }}(y)\right) \subset X$. Therefore, $x \in \bigcup_{\rho_{X}^{\text {ext }}(y)>r} B\left(y, \rho_{X}^{\text {ext }}(y)\right)$.

Now suppose that $x \in \bigcup_{\rho_{X}^{\text {ext }}(y)>r} B\left(y, \rho_{X}^{\text {ext }}(y)\right)$. Then $x \in B\left(y, \rho_{X}^{\text {ext }}(y)\right) \subset X$ for some $y$. Because $\rho_{X}^{\text {ext }}(y)>r$ it holds that $B\left(y, \rho_{X}^{\text {ext }}(y)\right)=\bar{B}\left(y, r_{0}\right)$ for some $r_{0} \geq r$. We now have $x \in$ $\bar{B}\left(y, r_{0}\right) \subset X$ for some $r_{0} \geq d$, so $x \in X \circ \bar{B}\left(r_{0}\right) \subset \bigcup_{s>r} X \circ \bar{B}(s)=\alpha_{r}(X)$.

The second part can be proven in the same way. It can also be deduced from the first part by observing that $\rho_{X}^{\text {int }}(x) \geq r \Leftrightarrow \rho_{X}^{\text {ext }}(x)>r$ and $\bar{B}\left(x, \rho_{X}^{\text {int }}(x)\right)=B\left(x, \rho_{X}^{\text {ext }}(x)\right)$.

From this theorem, an algorithm for computing size distributions can be derived. The algorithm can be used for chamfer metrics or any other metric for which distance transforms and reconstructions can be computed. Let $\theta_{r}$ be the function defined by

$$
\theta_{r}(s)= \begin{cases}0 & s \leq r \\ s & s>r\end{cases}
$$

3. Algorithm. Computation of the size distribution $\alpha_{r}$ of a bounded subset $X$ of $\mathbf{Z}^{2}$ with respect to a chamfer metric.

(1) Calculate the external distance transform $\rho_{X}^{\text {ext }}$.

(2) Remove values smaller than or equal to $r$ from the distance transform: let $f_{X}^{r}$ be the function $\theta_{r} \circ \rho_{X}^{\text {ext }}$.

(3) Calculate the reconstruction of $f_{X}^{r}$.

This algorithm requires five image scans: two for the calculation of the distance transform, one for the removal of small values and two for the reconstruction. In figure 6 , an example of the calculation of the opening transform is shown.

4. Definition. Let $E$ be some set and let $\Lambda$ be a totally ordered set. An anti-size distribution $[16,8]$ is a family $\left\{\phi_{\lambda}\right\}_{\lambda \in \Lambda}$ of operators mapping subsets of $E$ to subsets of $E$ such that for all $X, Y \subset E, r, s \in \Lambda$ :

(1) $X \subset Y \Rightarrow \phi_{r}(X) \subset \phi_{r}(Y)$.

(2) $X \subset \phi_{r}(X)$.

(3) $\phi_{r} \circ \phi_{r}=\phi_{r}$

(4) $\phi_{r} \circ \phi_{s}=\phi_{\max (r, s)}$.

The first three conditions are exactly the conditions used for defining that each $\phi_{\lambda}$ is a closing [16]. The fourth condition describes the composition behaviour of these closings. As with the definition of the size distribution, the third condition follows from the fourth. The index set $\Lambda$ will be $D$.

The goal is to construct an anti-size distribution based on spheres in the chamfer metric. Conceptually, size distributions and anti-size distributions are very related, because the anti-size distribution of an object is equivalent to the size distribution of its complement. This observation, however, does not lead to useful algorithms, because the complement of a bounded set is not bounded. Therefore the anti-size distribution is treated here separately.

Structural closings [14] are defined by

$$
X \cdot \bar{B}(r)=\bigcap_{X \subset \bar{B}_{h}^{C}} \bar{B}_{h}^{C} .
$$

This operation is illustrated in figure 5. The structural closing of $X$ with structuring element $B$ is found by fitting the structuring element in the background. If a point $x$ is contained in a translate $B_{h}$ of the structuring element which does not intersect $X, x$ is not a point of $X \bullet B$, otherwise it is. 


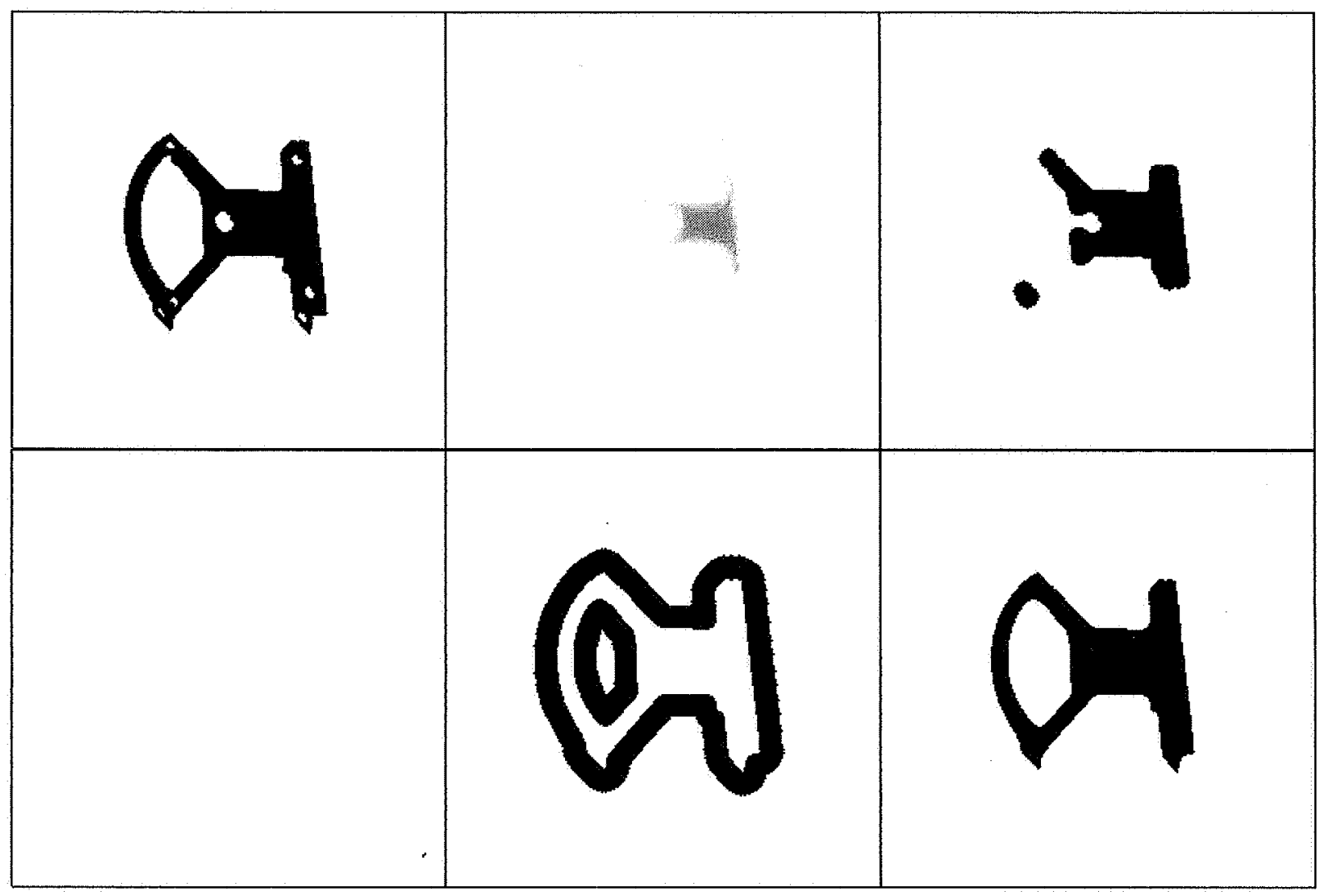

Figure 6. Image (b) shows the distance transform of an object (a) where small distance transform values have been removed. Dark pixels indicate large distance transform values. From this, the opening (c) is constructed. Figure (d) shows the distance transform of the background, with both small and large distance transform values having been removed. From the reconstruction (e) of this function, the closing $(f)$ is calculated.

As was the case with the structural opening and the size distribution, the structural closing satisfies the first three conditions, but not the fourth one. Analogously to the case of size distributions, a anti-size distribution can be defined by

$$
\phi_{r}=\bigcap_{s \geq r} X \cdot \bar{B}(s)
$$

Again, the anti-size distribution is not only useful for the analysis of sizes, but it also provides an alternative for the structural closing. The operation $\phi_{r}$ is the dual of the opening discussed above: $\phi_{r}(X)=\left[\alpha_{r}\left(X^{C}\right)\right]^{C}$. This duality suggests a way of calculating $\phi_{r}(X)$ : first calculate the complement of $X$; calculate the opening of this set with the algorithm presented above; take the complement of the result. The problem is that $X^{C}$ is not a bounded set. Therefore, this algorithm cannot be performed.

Fortunately, the algorithm can be adapted in such a way that the computation becomes finite. The algorithm for the p-q metric will be discussed. Analogous algorithms exist for other chamfer metrics.

5. Theorem. Let $X$ be a bounded subset of $\mathbf{Z}^{2}$, provided with the p-q-metric. Let $\rho_{X^{c}}$ be the distance transform of the background of $X$. Let $V$ be the set $\left\{x \in X \mid \rho_{X}^{\text {ext }} \leq r+q\right\}$. Let $1_{V}$ be the characteristic function of $V$. Then $\phi_{r}(X)=V \cap R\left(1_{V} \cdot \theta_{r} \circ \rho_{X^{c}}^{\text {ext }}\right)$. 
Proof. Write $\psi(X)$ for the set $V \cap R\left(1_{V} \cdot \theta_{r} \circ \rho_{X^{c}}\right)$. Let $x$ be a point in $\mathbf{Z}^{2}$. If $\rho_{X^{c}}^{\text {ext }}(x)>r+q$ then $x \notin V$, so $x \notin \psi(X)$. It also holds that $\bar{B}(x, r+q) \subset X^{C}$, so $x \notin X \bullet \bar{B}(r+q)$, so $x \notin \phi_{r}(X)$.

In the sequel it is assumed that $\rho_{X^{c}}^{\text {ext }}(x) \leq r+q$. It will be shown that $x \notin \phi_{r}(X) \Rightarrow x \notin \psi(X)$ and that $x \notin \psi(X) \Rightarrow x \notin \phi_{r}(X)$.

Suppose $x \notin \phi_{r}(X)$. Then there is a $y \notin X$ and an $s \geq r$ such that $x \in \bar{B}(y, s) \subset X^{C}$. From the second inclusion it follows that $r \leq s<\rho_{X^{c}}^{\text {ext }}(y)$. Two cases must be discerned: $\rho_{X^{c}}^{\text {ext }}(y) \leq r+q$ and $\rho_{X^{c}}^{\text {ext }}(y)>r+q$. If $\rho_{X^{c}}(y) \leq r+q$, then $1_{V}(y) \cdot \theta_{r}\left(\rho_{X^{c}}^{\text {ext }}(y)\right) \neq 0$. The point $y$ participates in the reconstruction step and $x \in B\left(y, \rho_{X^{c}}^{\text {ext }}(y)\right)$ is a point not contained in $\psi(X)$. If $\rho_{X^{c}}(y)>r+q$, let $P$ be a shortest path from $x$ to $y$. Let $z$ be the point on $P$ which is nearest to $y$ and satisfies $\rho_{X^{c}}(z) \leq r+q$. Due to the construction of the chamfer metric, this point satisfies $\rho_{X^{c}}^{\text {ext }}(z) \geq r$. Because $z$ lies on a shortest path from $x$ to $y$, we have $d(x, z)=d(x, y)-d(z, y)<\rho_{X^{c}}^{\text {ext }}(y)-d(z, y)$. From the triangle inequality it follows that $\rho_{X^{C}}^{\text {ext }}(z) \geq \rho_{X^{c}}^{\text {ext }}(y)-d(z, y)$. Therefore $d(x, z)<\rho_{X^{c}}(z)$. From $r \leq \rho_{X^{c}}(z) \leq r+q$ it follows that $1_{V}(z) \cdot \theta_{r} \rho_{X^{c}}(z) \neq 0$, so $x \in R\left(1_{V} \cdot \theta_{r} \rho_{X^{c}}\right)$. Therefore $x \notin \psi(X)$.

The second part of the proof goes as follows. Suppose that $x \notin \psi(X)$. Then there is an $y \in \mathbf{Z}^{2}$ such that $\rho_{X^{c}}^{\text {ext }}(y)>r$ and $x \in B\left(y, \rho_{X^{c}}^{\text {ext }}(y)\right)$. Therefore $x \notin X \bullet \bar{B}_{\rho_{X^{c}}^{\text {int }}(y)}$, so $x \notin \phi_{r}(X)$.

This theorem suggests the following algorithm for the computation of $\phi_{r}(X)$.

6. Algorithm. The computation of the anti-size distribution $\phi_{r}$ of a bounded subset $X$ of $\mathbf{Z}^{2}$ with respect to the $p$-q-metric.

(1) Calculate $\rho_{X c}^{\text {ext }}$ on a region which is large enough to contain $V$.

(2) Remove all points from the distance transform having a value smaller than or equal to $r$.

(3) Remove all points having a distance transform larger than $r+q$ as well.

(4) Calculate the reconstruction of the remaining function.

(5) Take the intersection of the complement of the reconstructed set with the set of points $x$ with $\rho_{X^{c}}^{\text {ext }}(x) \leq r+q$.

The execution of this algorithm requires five scans of a somewhat enlarged region, because the region must be large enough to contain $V$.

This theorem can also be interpreted in the following way: in order to compute the anti-size distribution, which is defined as an intersection of an infinite number of sets, it is sufficient to perform finitely many - at most $q$-structural closings and compute their intersection. Likewise, it can be seen that size distributions can be computed as the union of at most $q$ structural openings. This observation, however, does not provide an efficient algorithm, because there is no fast algorithm for performing structural openings with spheres as structuring elements.

Figure 6 shows an example of the calculation of the anti-size distribution.

\section{The Opening Transform}

In section 6 , the size distribution $\alpha_{r}$ has been presented. In multi scale analysis, it is often necessary to calculate $\alpha_{r}$ for all possible values of $r$. The fact that $\alpha_{r}(X) \subset \alpha_{s}(X)$ for $r \geq s$ makes it possible to define the opening transform:

1. Definition. Let $d$ me a discrete metric on a set $E$ and let $D$ be its range. Let $X$ be a bounded subset of $E$. The opening transform $A_{X}$ is the function $E \rightarrow D$ such that

$$
A_{X}(x)=\max \left\{r \in D \mid x \in \alpha_{r}(X)\right\} .
$$

The sets $\alpha_{r}(X)$ can be calculated for different values of $r$ by a simple thresholding of $A_{X}$. The object $X$ can be analysed by comparing the images $\alpha_{r}(X)$ for subsequent values of $r$. For example, 
the function which maps $r$ to the number of pixels in $\alpha_{r}(X) \backslash \alpha_{r+1}(X)$ is called the pattern spectrum [8]. Note that the pattern spectrum of $X$ is equal to the histogram of $A_{X}$.

The straightforward way of computing the opening transform would be to calculate $\alpha_{r}(X)$ for all values of $r$ up to the largest value of the internal distance transform of $X$. If one starts with the distance transform of $X$, this requires three image scans per level: one for the removal of small values of the distance transform and two for the reconstruction. The information from all $\alpha_{r}(X)$ is combined in order to find the opening transform.

2. Algorithm. Opening transform of a bounded subset $X$ of $\mathbf{Z}^{2}$ with respect to a chamfer metric (brute force).

(1) Give all pixels in the result image value 0.

(2) Compute the external distance transform of $X$.

(3) For all radii $r$ up to the largest value of the distance transform of $X$ :

- remove all values smaller than $r$ from the distance transform;

- perform a reconstruction of the remaining function;

- give all points in the reconstructed set the value $r$ in the result image.

In most cases, the costs of this algorithm are prohibitively large. Using the medial axis and an alternative way for performing reconstructions, a more efficient algorithm can be constructed.

3. Theorem. Let $d$ be a discrete metric on a set $E$ and let $D$ be its range. Let $X$ be a bounded subset of $E$ and let $M_{X}$ be its medial axis. Let $r \in D$. Then

$$
\alpha_{r}(X)=\bigcup\left\{\bar{B}\left(m, \rho_{X}^{\mathrm{int}}(m)\right) \mid m \in M_{X} ; \rho_{X}^{\mathrm{int}}(m) \geq r\right\}
$$

Proof. Suppose that $x \in \alpha_{r}(X)$. Then there is a $s \geq r, y \in X$ such that $x \in \bar{B}(y, s) \subset X$. By construction of the medial axis, there is a $m \in M_{X}$ such that $\bar{B}(y, s) \subset \bar{B}\left(m, \rho_{X}^{\text {int }}(m)\right) \subset X$. From this, it follows that $\rho_{X}^{\text {int }}(m) \geq s \geq r$. Therefore $x \in \bigcup\left\{\bar{B}\left(m, \rho_{X}^{\text {int }}(m)\right) \mid m \in M_{X} ; \rho_{X}^{\text {int }}(m) \geq r\right\}$.

Now suppose that $x \in \bigcup\left\{\bar{B}\left(m, \rho_{X}^{\text {int }}(m)\right) \mid m \in M_{X} ; \rho_{X}^{\text {int }}(m) \geq d\right\}$ Then $x \in \bar{B}(m, s) \subset X$ for some $s \geq r$, so $x \in X \circ \bar{B}(r) \subset \alpha_{r}(X)$.

From this theorem it follows that $A_{X}(x)=r$ if $x \in \bigcup\left\{\bar{B}(m, r) \mid m \in M_{X} ; \rho_{X}^{\text {int }}(m)=r\right\}$ and $x \notin \bigcup\left\{\vec{B}(m, s) \mid m \in M_{X} ; \rho_{X}^{\text {int }}(m)=s\right\}$ for all $s>r$. For $\mathrm{p}$-q-metrics, the opening transform can be computed using the following

4. Algorithm. Opening transform of a bounded subset $X$ of $\mathbf{Z}^{2}$ with respect to the p-q-metric by reconstruction on ordered medial axis points.

(1) Set all pixels in the result image to 0.

(2) Compute the internal distance transform of $X$.

(3) Detect the medial axis points and sort them in order of increasing distance transform value.

(4) For all medial axis points $x$, in order of increasing distance transform value: set all pixels in $\bar{B}\left(x, \rho_{X}^{\mathrm{int}}(x)\right)$ in the result image to $r$.

This algorithm requires computation of distance transforms and reconstructions. The third step requires the computation of the medial axis. Therefore, the algorithm is only suited for p-q-metrics. Because the medial axis points have integer distance transform values and the number of occurring distance transform values is in general smaller than the number of medial axis points, the sorting in step (3) can be done quickly using distribution sorting [7]. The last step is performed using a precalculated table of offsets of pixels in a sphere with respect to the center of this sphere. Thus it is not necessary to access the whole image for each iteration in step (4). Each pixel in a sphere can be accessed directly. This method of addressing, together with the fact that the number of 


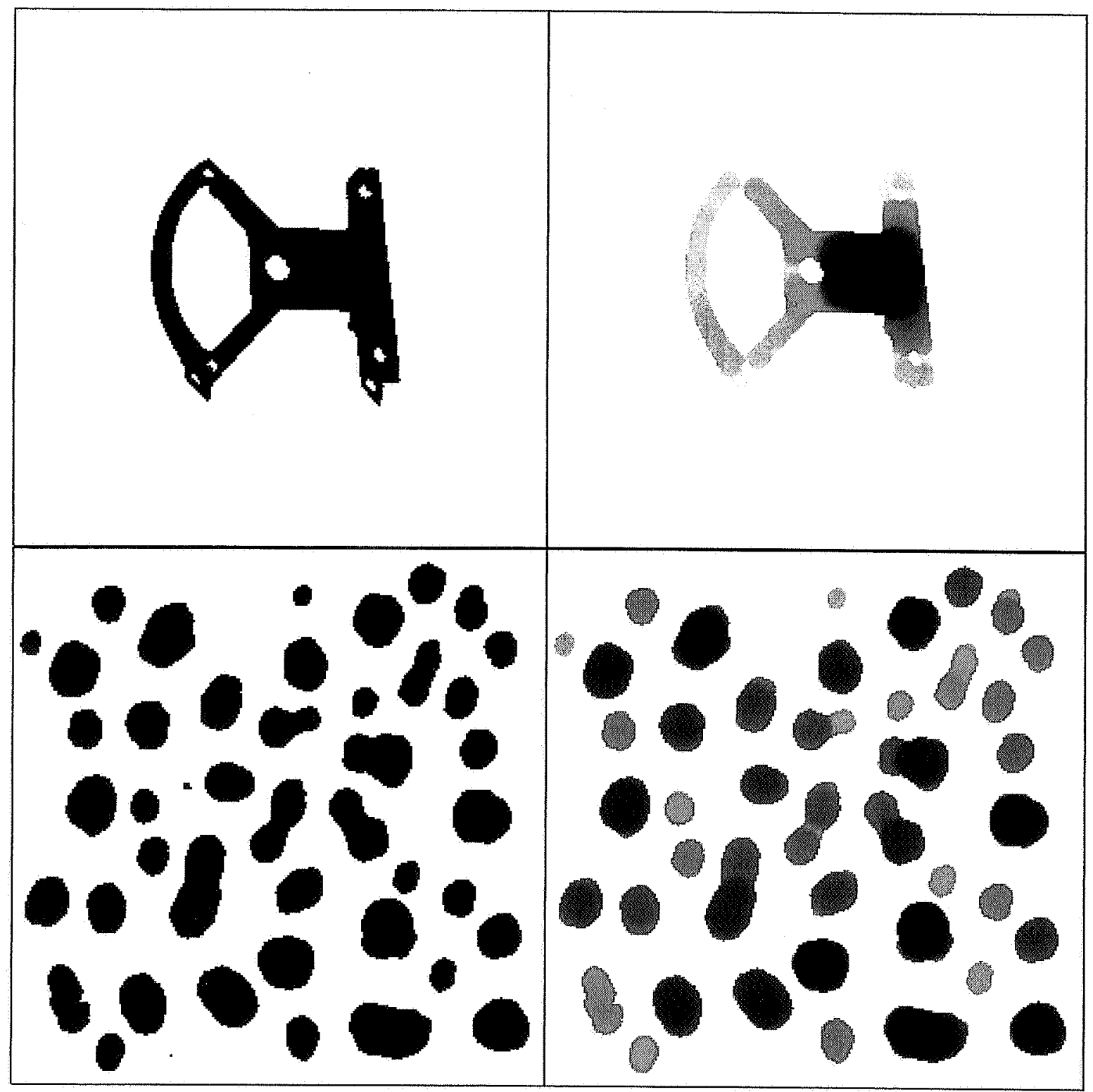

Figure 7. Two binary images $(a, c)$ and their opening transforms $(b, d)$. Dark pixels indicate large values for the opening transform.

medial axis points is small compared with the total number of pixels in the image, accounts for the efficiency of this algorithm. The addressing technique is described in detail in the appendix.

The run time of this algorithm depends on the shape of the image, because the number of point insertions to be performed, varies. Figure 7 shows some binary images and their opening transform. For the first image, the brute force algorithm takes $18.70 \mathrm{~s}$., while reconstruction on ordered medial axis points takes $0.17 \mathrm{~s}$.; for the second image, the brute force algorithm takes 11.07 $\mathrm{s}$. and reconstruction on ordered medial axis points takes $0.22 \mathrm{~s}$. Both algorithms use a distance transformed image as an input. The image sizes are $256 \times 256$ and the algorithm runs on a Silicon Graphics Indigo workstation. It can be seen that reconstruction on ordered medial axis points is 50 to 100 times faster than the brute force algorithm. 


\section{Conclusions}

This paper has presented an integration of chamfer metrics and mathematical morphology. Both theoretical aspects and efficient algorithms have been discussed. As chamfer metrics on a square grid can approximate the Euclidean metric within an error of a few percent, morphological operations based on chamfer metrics such as the 5-7-metric or the 5-7-11 metric are a good approximation of morphological operations using Euclidean discs. They perform much better that the conventional operators using square or diamond shaped structuring elements. The use of operations based on chamfer metrics also gives improved rotation invariance, compared with conventional methods.

First some properties of chamfer metrics were derived. It has been shown, which values can be assumed by a chamfer metric and which paths can occur as a shortest path between two points. For this purpose, the notions of adjacent prime vectors, normalised prime vectors and wedges have been defined.

The medial axis is defined as the center of maximal spheres with respect to a chamfer metric. From this medial axis, the original object can be reconstructed when the values of the distance transform in the medial axis points are given. It has been shown how the centers of maximal spheres for the p-q-metric can be computed efficiently using the distance transform. An algorithm is presented which requires only three image scans and is based on local operations only. The computation of the medial axis associated with the p-q-r-metric is a subject of ongoing research.

From the medial axis, a medial line has been derived. This medial line can be derived from the medial axis using thinning, as described by Hilditch; such a thinning provably yields a homotopy preserving medial line. It is conjectured that a second algorithm using path climbing also computes a homotopy preserving medial line.

A size distribution and an anti-size distribution have been defined using chamfer metrics. The investigation of the relation between (anti-)size distributions, distance transformations and reconstruction has lead to an efficient algorithm for the computation of these operators. The algorithm requires four image scans and is based on local operation only.

Based on size distributions associated with chamfer metrics, the opening transform is defined. An investigation of the relation between the opening transform, the medial axis, distance transform and reconstructions has lead to an algorithm for the computation of the opening transform which is 50 to 100 times faster than the brute force method. If the 5-7-chamfer metric is used in this algorithm, the resulting opening transform yields an accurate approximation of the opening transform based on the Euclidean metric. The pattern spectrum of an object can be derived from the opening transform by histogramming. The medial axis algorithm presented in this paper does not work for the 5-7-11 metric, as this metric is not extending. A medial axis algorithm for this metric is a subject of ongoing research.

Summarising, the integration of chamfer metrics into mathematical morphology presented in this paper has lead to a number of morphological operators which approximate morphological operations based on Euclidean discs accurately and which can be calculated efficiently.

\section{Acknowledgements}

The author thanks H. Heijmans, F. Groen and A. Toet for useful discussions on the subject and thorough reading of the manuscript. 
[1] C. Arcelli and G. Sanniti di Baja. A width-independent fast thinning algorithm. IEEE Transactions on Pattern Analysis and Machine Intelligence, 7(4):463-474, 1985.

[2] C. Arcelli and G. Sanniti di Baja. Finding local maxima in a pseudo-euclidean distance transform. Computer Vision, Graphics and Image Processing, 43:361-367, 1988.

[3] G. Borgefors. Distance transformations in arbitrary dimensions. Computer Vision, Graphics and Image Processing, 27:321-345, 1984.

[4] L. Dorst. Pseudo-euclidean skeletons. In Proceedings of the Eighth International Conference on Pattern Recognition, 1989.

[5] F. Groen and N. Foster. A fast algorithm for cellular logic operations on sequantial machines. Pattern Recognition Letters, 2:333-338, 1984.

[6] C. Hilditch. Linear skeletons from square cupboards. In B. Meltzer and D. Mitchie, editors, Machine Intelligence 4. University Press, Edinburgh, 1969.

[7] D. Knuth. The Art of Computer Programming Volume 3: Sorting and Searching. AddisonWesley, Reading Mass., U.S.A., 1973.

[8] P. Maragos. Pattern spectrum and multiscale shape representation. IEEE Transactions on Pattern Analysis and Machine Intelligence, 11(7):701-715, 1989.

[9] G. Matheron. Random Sets and Integral Geometry. Wiley, New York, 1975.

[10] G. Matheron. Examples of topological properties of skeletons. In J. Serra, editor, Image Analysis and Mathematical Morphology Volume 2: Theoretical Advances. Academic Press, London, 1988.

[11] F. Meyer. Skeletons and perceptual graphs. Signal Processing, 16:335-363, 1989.

[12] A. Montanvert. Medial line: Graph representation and shape description. In Proceedings of the Eighth International Conference on Pattern Recognition, 1986.

[13] C. Niblack, D. Capson, and P. Gibbobs. Generating skeletons and centerlines from the medial axis transform. In Proceedings of the Tenth International Conference on Pattern Recognition, 1990.

[14] C. Ronse and H. Heijmans. The algebraic basis of mathematical morphology part 2: Openings and closings. CVGIP Image Understanding, 54(1):74-97, 1991.

[15] M. Schmitt and L. Vincent. Morphology: Algorithms and Applications. Cambridge University Press, to appear.

[16] J. Serra. Image Analysis and Mathematical Morphology. Academic Press, London, 1982.

[17] B. Stewart. Theory of Numbers. The Macmillan Company, 1964.

[18] R. van den Boomgaard and R. van Balen. Methods for fast morphological image transformations using bitmapped binary images. CVGIP Graphical Models and Image Processing, 54(3):252-258, 1992.

[19] B. Verwer. Local distances for distance transformations in two and three dimensions. Pattern Recognition Letters, 12(11):671-682, 1991.

[20] L. Vincent. Algorithmes Morphologiques a Base de Files d'Attente et de Lacets. PhD thesis, Ecole Nationale Superieure des Mines de Paris, Fontainebleau, 1990. 


\section{A. Appendix.}

This appendix describes the addressing technique which is used in the fast opening transform algorithm described in section 7 . In this algorithm, all pixels in a sphere $\bar{B}(x, r)$ must be accessed and their value must be set to $r$. Using a precalculated offset table, this can be done very efficiently. The examples in this appendix are based on the 5-7-metric, but the technique illustrated here can be used for all chamfer metrics.

The image is stored in an array image of length $w \times h$, where $w$ and $h$ are the image width and height, respectively. If the upper left hand corner of the image has coordinates $(0,0)$ and the $x$ and $y$-coordinates increase towards the right and downwards, respectively, the value of pixel $(x, y)$ is stored in the $(x+w \times y)$-th entry of image.

Let $(x, y)$ be a pixel with offset $i$. Then the pixel $(x+\delta x, y+\delta y)$ has offset $i+(\delta x+w \times \delta y)$. The number $\delta x+w \times \delta y$ is called the relative offset of $(x+\delta x, y+\delta y)$ with respect to $(x, y)$.

The algorithm uses a precalculated offset table offset in order to store the relative offsets of the points in a sphere with respect to the center of the sphere. This is illustrated in figure 8 . The first entry in offset is 0 , the relative offset of the center of the sphere with respect to itself. The next four entries are the relative offsets of the points at distance 5 from the center. The relative coordinates of these points are $(1,0),(0,1),(-1,0)$ and $(0,-1)$ and the corresponding relative offsets are $+1,-w,-1$ and $+w$. The next four entries are the relative offsets of the points at distance 7 from the center, et cetera. The array last-offset contains as its $r$-th entry the index of the last relative offset in off set which corresponds to a pixel at distance $r$ from the center of the sphere.

Now let $i$ be the offset of some point $x$ in the image. Then the points in $\bar{B}(x, r)$ are the points with offsets $i+$ offset[0], $i+$ offset[1], ..,i offset[last-offset[r]]. Thus, setting all pixels in $\bar{B}(x, r)$ to $r$ can be done using the loop

$$
\begin{gathered}
\text { for } j=0 \text { to last-index }[\mathrm{r}] \\
\text { image }[\mathrm{i}+\text { offset }[\mathrm{j}]]=\mathrm{r}
\end{gathered}
$$

The actual programme written by the author is written in C. This language has pointer arithmetic, which allows some further optimisations of the addressing scheme. 

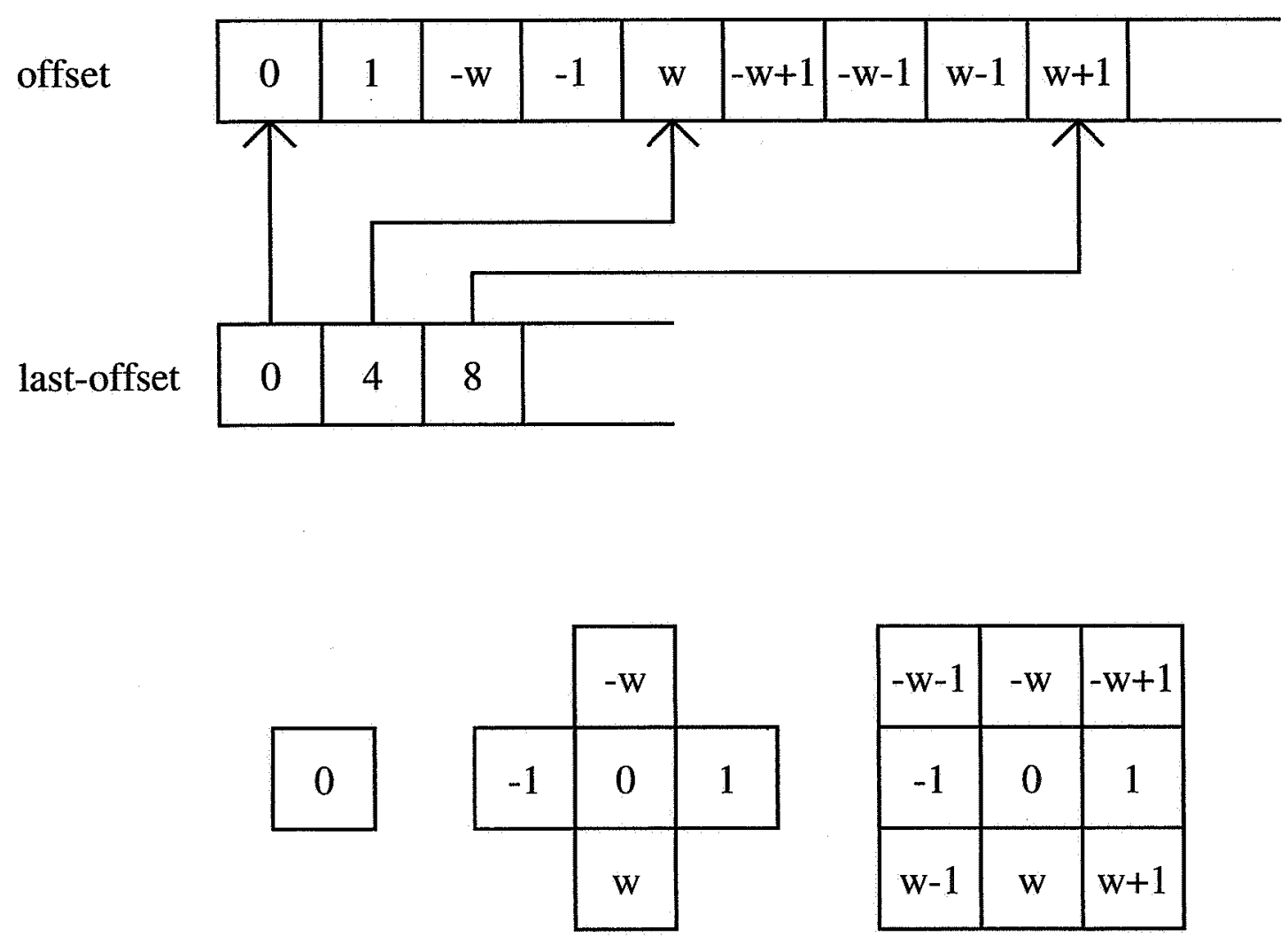

The beginning parts of the tables offset and last-offset. The closed spheres of radii 0,5 and 7 are shown, and the relative offsets of pixels with respect to the center are shown (w is the width of the grid). 